Gut and Liver, Vol. 11, No. 6, November 2017, pp. 835-842

\title{
Healthcare Costs for Chronic Hepatitis C in South Korea from 2009 to 2013: An Analysis of the National Health Insurance Claims' Data
}

\author{
Moran $\mathrm{Ki}^{1}$, Hwa Young $\mathrm{Choi}^{1}$, Kyung-Ah $\mathrm{Kim}^{2}$, Eun Sun Jang ${ }^{3}$, and Sook-Hyang Jeong ${ }^{3}$ \\ ${ }^{1}$ Department of Cancer Control and Policy, Graduate School of Cancer Science and Policy, National Cancer Center, ${ }^{2}$ Department of Internal \\ Medicine, Inje University Ilsan Paik Hospital, Goyang, and ${ }^{3}$ Department of Internal Medicine, Seoul National University Bundang Hospital, \\ Seoul National University College of Medicine, Seongnam, Korea
}

\section{See editorial on page 745 .}

Background/Aims: The introduction of direct-acting antivirals (DAA) in 2013 revolutionized hepatitis C virus (HCV) treatment, offering a cure rate $>90 \%$. However, this therapy is expensive, and estimations of the number of chronic HCVinfected $(\mathrm{CHC})$ patients and their treatment costs pre-2013 are therefore essential for creating policies and expanding drug access. Herein, we aimed to investigate the number of HCV-related liver disease patients, their healthcare utilization, their annual direct medical costs, and the interferonbased antiviral treatment rates and costs from 2009 to 2013 in South Korea. Methods: The National Health Insurance database was reviewed, and patients diagnosed with CHC from 2009 to 2013 were extracted. Data regarding detailed healthcare utilization, prescribed drugs, and direct medical costs were obtained. For annual direct healthcare cost calculations, a prevalence-based approach was used. Results: Overall, 181,768 CHC patients were identified. In 2013, the annual per-patient costs for chronic hepatitis, liver cirrhosis, hepatocellular carcinoma, and the first year post-liver transplant were 895, 1,873, 6,945, and 67,359 United States dollars, respectively. Interferon-based antiviral therapeutics were prescribed to 25,223 patients (13.9\%). Conclusions: Healthcare costs have increased remarkably with increasing liver disease severity. Thus, efforts to stop disease progression are needed. Moreover, the low rate of interferon-based therapy indicates an unmet need for DAA.

(Gut Liver 2017;11:835-842)
Key Words: Antiviral agents; Costs and cost analysis; Hepatitis C, chronic; Pegylated interferon; Ribavirin

\section{INTRODUCTION}

Hepatitis C virus (HCV) is a leading cause of liver cirrhosis and hepatocellular carcinoma (HCC). Worldwide, approximately 80 million people have chronic HCV infection (CHC), corresponding to a global prevalence of $1.1 \%$, and about 700,000 people with $\mathrm{CHC}$ die untreated annually. ${ }^{1}$ The majority of $\mathrm{CHC}$ patients come from the Western Pacific and Southeast Asia regions; however, HCV infection is an understudied health problem in many Asian countries, perhaps due to being overshadowed by the more prevalent hepatitis B virus infection. ${ }^{2}$

Despite its low efficacy and high rate of adverse events, interferon-based antiviral therapy has long been considered a standard therapy for HCV infection. However, a greater understanding of the HCV genome and life cycle led to the introduction of direct-acting antivirals (DAAs) in 2013, which brought a revolutionary change in HCV therapeutics. An 8- to 24-week treatment with DAAs has been reported to result in cure in more than 90\% of the patients, with fewer adverse events, opening a new horizon to HCV eradication. However, the very high cost of DAA therapy is a barrier to the treatment access and places a heavy burden on the health systems in many resource-constrained countries.

Therefore, estimation of the nationwide number of $\mathrm{CHC}$ patients and the medical costs for their treatment before the DAA era is an essential step for creating policies to promote expanded access to DAA therapy. The National Health Insurance (NHI), run by the Korean government, is a nationwide universal

\section{Correspondence to: Sook-Hyang Jeong}

Department of Internal Medicine, Seoul National University Bundang Hospital, Seoul National University College of Medicine, 82 Gumi-ro 173 beon-gil, Bundang-gu, Seongnam 13620, Korea

Tel: +82-31-787-7029, Fax: +82-31-787-4052, E-mail: jsh@snubh.org

Received on January 17, 2017. Revised on March 6, 2017. Accepted on March 6, 2017. Published online August 14,2017

pISSN 1976-2283 eISSN 2005-1212 https://doi.org/10.5009/gnl17034

Moran Ki and Hwa Young Choi contributed equally to this work as first authors.

@ This is an Open Access article distributed under the terms of the Creative Commons Attribution Non-Commercial License (http://creativecommons.org/licenses/by-nc/4.0) which permits unrestricted non-commercial use, distribution, and reproduction in any medium, provided the original work is properly cited. 
insurance system, which covered 97\% of the total population in 2010, and all inpatient and outpatient healthcare utilization data are available in the Health Insurance Review and Assessment Service (HIRA). ${ }^{3}$ Using the HIRA claims data, the present study aimed to investigate; (1) the nationwide number and healthcare utilization of the patients who made claims with diagnoses of HCV-related liver diseases; (2) their annual direct medical costs; and (3) the interferon-based antiviral treatment rates and costs for the HCV-associated liver diseases, from 2009 to 2013, before the introduction of DAA therapy in South Korea.

\section{MATERIALS AND METHODS}

\section{Data sources}

Data from the HIRA claims database from 2009 to 2013 were used for this study, which can be considered as representative data of the entire Korean population, because the NHI covered $97 \%$ of the total Korean population in 2010. The health insurance claims database can be divided into four categories: general specifications (basic demographic information of the patients, number of outpatient visits, and duration of inpatients hospitalization), health services (types of services, frequency, the itemized unit costs, inpatient medications, and medical materials), diagnosis information (history of all diseases that the patients were treated for), and detailed outpatient prescriptions (detailed information on outpatient medication dispensed at community pharmacies, including the dose, frequency, and cost). ${ }^{3}$ During the study period (2009 to 2013), pegylated interferon- $\alpha$ and ribavirin were the standard HCV therapeutics in Korea, because the first interferon-free DAA therapy was not introduced until 2015.

\section{Patient selection and diagnostic classification}

All patients who had ever been diagnosed with CHC (International Statistical Classification of Diseases and Related Health Problems 10th Revision [ICD-10] code: B18.2), either as a primary or secondary diagnosis, were selected based on the health insurance claims data from 2009 to 2013. Among them, patients with liver cirrhosis, HCC, and liver transplantation (LT) were defined as those who were diagnosed with liver cirrhosis (ICD-10 codes: K74, fibrosis and cirrhosis of liver; K74.0, hepatic fibrosis; K74.1, hepatic sclerosis; K74.2, hepatic fibrosis with hepatic sclerosis; K74.6, other and unspecified cirrhosis of liver), HCC (C22, malignant neoplasm of liver and intrahepatic bile ducts; C22.0, liver cell carcinoma; C22.9, liver, unspecified), and LT (Z94.4, liver transplant status; T86.4, liver transplant failure and rejection), respectively, after at least 1 year following the diagnosis of HCV infection (B18.2, chronic viral hepatitis C). For LT patients, those who had both a diagnostic code of LT and a specific claim code for LT surgery were included. For the cost of LT, it was categorized as the cost within and after 1 year from the surgery, considering the high cost of the operation. If the selected patients had more than two diagnostic codes besides the codes mentioned above, the most severe disease code was designated to each patient; the severity of liver disease was categorized, from mildest to the most severe, as chronic hepatitis, liver cirrhosis, HCC, and LT. Patients with a diagnostic code of hepatitis B virus or HIV infection were excluded.

The study protocol was approved by the Institutional Review Board of the Seoul National University Bundang Hospital (X1312/229-906).

\section{Data retrieval on healthcare utilization, prescribed drugs, and medical cost}

All data regarding detailed healthcare utilization, prescribed drugs, and direct medical costs were obtained from the abovementioned three categories of "general specifications," "health services," and "outpatient prescriptions." Five different antiviral drugs against HCV were prescribed during the study period, including interferon- $\alpha-2 a$, interferon- $\alpha-2 b$, peginterferon- $\alpha-2 a$, peginterferon- $\alpha-2 b$, and ribavirin. For the analysis, the patients were divided into the monotherapy group, which included cases to whom only one drug was prescribed, and the combination therapy group, which included cases prescribed two or more antiviral drugs among the above five drugs.

For calculation of the annual direct healthcare costs for HCVrelated liver diseases from 2009 to 2013, a prevalence-, rather than incidence-based, approach was used, owing to the short study period of only 5 years. The medical costs calculated by the incidence-based approach includes the total costs over one's entire life span or a considerably long period, while the prevalence-based approach includes a total yearly cost paid for newly diagnosed patients and preexisting patients each year. ${ }^{4}$ The currency exchange rate was determined by rounding down the average exchange rate of 1 United States dollar (USD) to 1,152.168 Korean Won between 2009 and 2013; therefore, 1 USD equals 1,152 Korean Won in this study. Since the HIRA claims database does not contain data on the medical costs that are not covered by the insurance (such as certain ultrasound services or drugs) or nonhealthcare costs (such as transportation, household expenditures, relocation, property losses, and informal care), this study only included direct healthcare costs covered by the NHI, regardless of who paid (i.e., paid by both NHI and patient).

\section{Statistical analysis}

All dependent and independent variables were analyzed descriptively, and the numbers and proportions are reported. Continuous variables are presented as the mean and standard deviation for normally distributed data, and as the median and interquartile range for non-normally distributed data. All statistical analyses were performed using SAS 9.3 (SAS Institute, Cary, NC, USA). 


\section{RESULTS}

\section{Demographic characteristics and healthcare costs of patients with CHC from 2009 to 2013}

The nationwide number of patients who made claims with the diagnosis of CHC from 2009 to 2013 was 181,768, with similar sex distributions (51.9\% males, 48.2\% females) and a

Table 1. Demographic Features of Patients with Chronic Hepatitis C Virus Infection* in South Korea from 2009 to 2013: National Health Insurance Claims' Data

\begin{tabular}{lccc}
\hline Age, yr & Male & Female & Total \\
\hline $0-9$ & $622(0.7)$ & $481(0.6)$ & $1,103(0.6)$ \\
$10-19$ & $2,143(2.3)$ & $1,040(1.2)$ & $3,183(1.8)$ \\
$20-29$ & $5,056(5.4)$ & $4,260(4.9)$ & $9,316(5.1)$ \\
$30-39$ & $10,631(11.3)$ & $9,073(10.4)$ & $19,704(10.8)$ \\
$40-49$ & $21,270(22.5)$ & $14,675(16.8)$ & $35,945(19.8)$ \\
$50-59$ & $22,974(24.3)$ & $22,292(25.5)$ & $45,266(24.9)$ \\
$60-69$ & $18,113(19.2)$ & $19,254(22.0)$ & $37,367(20.6)$ \\
$70-79$ & $11,199(11.9)$ & $13,148(15.0)$ & $24,347(13.4)$ \\
$\geq 80$ & $2,368(2.5)$ & $3,169(3.6)$ & $5,537(3.0)$ \\
Total & $94,376(100)$ & $87,392(100)$ & $181,768(100)$ \\
Mean & 52.2 & 54.8 & 53.4 \\
\hline
\end{tabular}

Data are presented as number $(\%)$.

*Number of patients who received treatment for chronic hepatitis C virus infection (ICD 10-code: B18.2) from 2009 to 2013. mean age of 53.4 years (Table 1). Though the annual number of CHC patients increased from 65,321 in 2009 to 68,499 in 2013, the crude prevalence, calculated as the case number divided by the registered population at the end of the year, did not significantly differ during the 5-year period, at $0.131 \%$ in 2009 and $0.134 \%$ in 2013 (Fig. 1).

Over the 5 years, approximately 70\% of all CHC patients sought for medical evaluation. Of those patients, 36.4\% visited

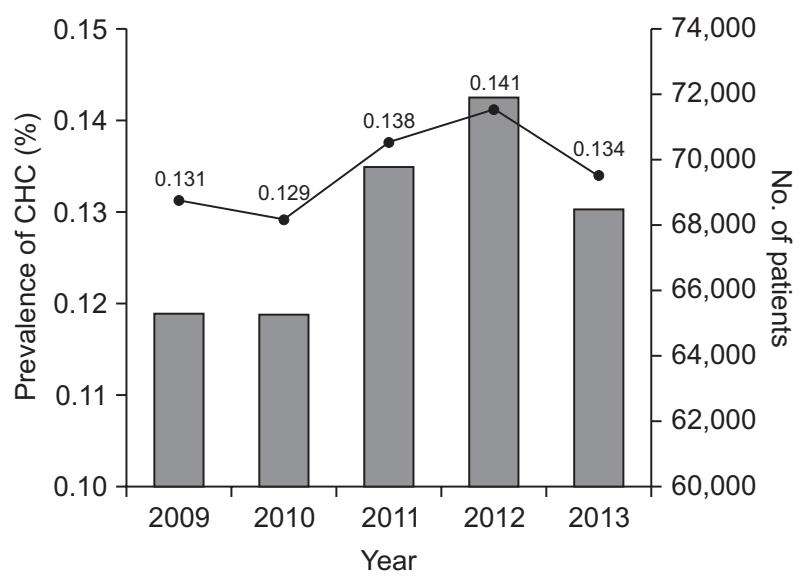

Fig. 1. The crude prevalence of chronic hepatitis $C$ virus infection (CHC) by year in South Korea. The line graph shows the prevalence rate, and the bar graph shows the number of patients.

Table 2. Healthcare Costs Covered by the National Health Insurance for Chronic Hepatitis C Virus Infection in South Korea from 2009 to 2013

\begin{tabular}{|c|c|c|c|c|c|c|c|}
\hline \multirow[b]{2}{*}{ Variable } & \multirow[b]{2}{*}{$\begin{array}{c}\text { No. of } \\
\text { patients (\%) }\end{array}$} & \multicolumn{3}{|c|}{ Hospital } & \multirow{2}{*}{$\begin{array}{c}\text { Pharmacy } \\
\text { Extra-hospital } \\
\text { pharmaceutical } \\
\text { cost, million USD }\end{array}$} & \multirow{2}{*}{$\begin{array}{c}\text { Total } \\
\text { healthcare cost, } \\
\text { million USD }\end{array}$} & \multirow{2}{*}{$\begin{array}{l}\text { Healthcare } \\
\text { cost per } \\
\text { patient, USD }\end{array}$} \\
\hline & & $\begin{array}{l}\text { Insurer payment } \\
\text { +copayment, } \\
\text { million USD }\end{array}$ & $\begin{array}{l}\text { Copayment, } \\
\text { million USD }\end{array}$ & $\begin{array}{l}\text { Proportion of } \\
\text { copayment, \% }\end{array}$ & & & \\
\hline \multicolumn{8}{|l|}{ Healthcare facilities* } \\
\hline Tertiary hospital & $80,729(36.4)$ & 123.19 & 44.77 & 36.3 & 21.40 & 144.59 & 1,791 \\
\hline General hospital & $56,358(25.4)$ & 100.78 & 28.03 & 27.8 & 16.53 & 117.30 & 2,081 \\
\hline Hospital & $17,603(7.9)$ & 19.37 & 4.48 & 23.1 & 2.77 & 22.15 & 1,258 \\
\hline Primary clinic & $65,404(29.5)$ & 22.94 & 4.55 & 19.8 & 18.44 & 41.38 & 633 \\
\hline Geriatric center & $1,314(0.7)$ & 3.68 & 0.61 & 16.6 & 0.07 & 3.74 & 2,847 \\
\hline Community health center & $578(0.3)$ & 0.05 & 0.01 & 21.6 & 0.15 & 0.20 & 349 \\
\hline Total & $221,986(100)^{\dagger}$ & 270 & 82 & 30.5 & 59 & 329 & $1,812^{\ddagger}$ \\
\hline \multicolumn{8}{|l|}{ Year } \\
\hline 2009 & 65,321 (19.2) & 50 & 15 & 30.2 & 12 & 62 & 948 \\
\hline 2010 & $65,245(19.1)$ & 51 & 16 & 30.9 & 12 & 63 & 969 \\
\hline 2011 & $69,773(20.5)$ & 55 & 17 & 31.0 & 12 & 67 & 960 \\
\hline 2012 & $71,918(21.1)$ & 57 & 17 & 30.5 & 12 & 69 & 958 \\
\hline 2013 & $68,499(20.1)$ & 56 & 18 & 30.0 & 12 & 68 & 997 \\
\hline Total & $340,756(100)^{\S}$ & 270 & 82 & 30.5 & 59 & 329 & $1,812^{\S}$ \\
\hline
\end{tabular}

USD, United States dollar (1 USD=1,152 South Korean Won).

*Tertiary hospitals are teaching hospitals specializing in the treatment of severe diseases. General hospitals are secondary level healthcare facilities for inpatients with more than 100 beds. Hospitals are primary level healthcare facilities for inpatients with more than 30 beds; ${ }^{\dagger}$ The number of healthcare facility visits may overlap for each patient; ${ }^{\dagger}$ Healthcare cost per patient=total healthcare cost from 2009 to 2013 (329 million USD)/ number of individual patients who received treatment for chronic hepatitis C virus infection from 2009 to 2013 (181,768); ${ }^{\circ}$ The number of patients who received treatment in each year may overlap. The number of individual patients with chronic hepatitis $\mathrm{C}$ virus infection during the 5-year study period was 181,768 . 


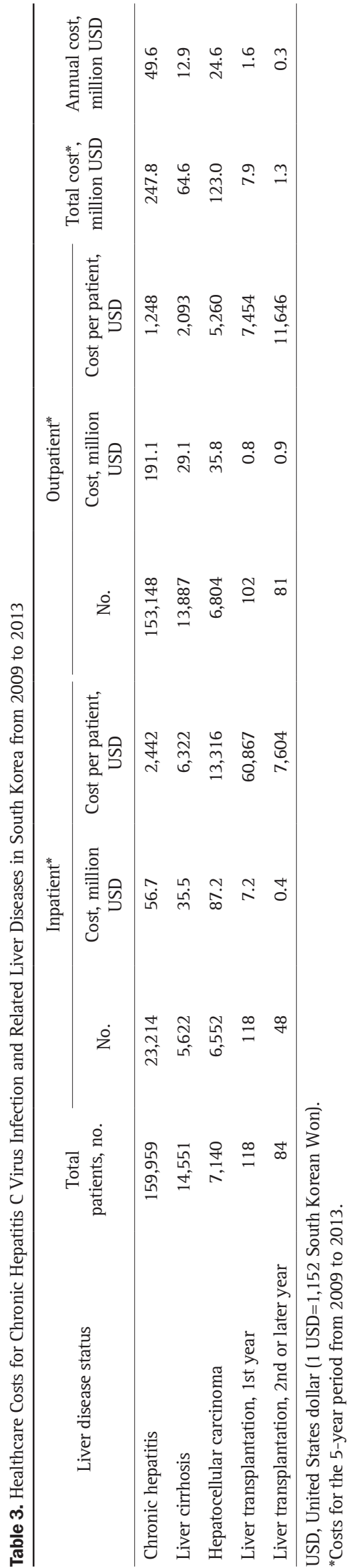

a tertiary hospital, 25.4\% visited general hospital, 7.9\% visited hospital, and 29.5\% visited a primary clinic. The total health care cost during the 5 years was 329 million USD, which included 283 million USD in hospital costs and 59 million USD for pharmacy costs. The hospital cost by year showed an increasing trend, from 50 million USD in 2009 to 56 million USD in 2013, while the annual pharmacy-related costs remained unchanged during the study period, at 12 million USD. Among the hospital costs, the proportion of copayment was 30.5\%. The healthcare cost per patient for each year was approximately 960 USD (948 to 997), while the total healthcare cost per patient during 5 years was 1,812 USD (Table 2).

\section{Healthcare costs for patients with $\mathrm{CHC}$ and related liver diseases from 2009 to 2013}

The direct healthcare costs covered by the NHI according to the liver disease severity and healthcare utilization (inpatient and outpatient care) during 2009 to 2013 are summarized in Table 3. All patients with related liver diseases (liver cirrhosis, HCC, and LT) had a CHC history in addition to these diseases. The total number of patients with CHC from 2009 to 2013 was 181,768 persons, including 31,762 inpatients (17.5\%), who were hospitalized at least once, and 173,109 outpatients (95.2\%) during the study period.

The number of patients with chronic hepatitis was 159,959, including 23,214 inpatients (14.5\%), who were hospitalized at least once, and 153,148 outpatients (95.7\%). The overall 5-year cost for treatment for chronic hepatitis was 247.8 million USD, which included inpatient and outpatient costs of 56.7 million USD (22.9\%) and 191.1 million USD (77.1\%), respectively. The annual cost was 49.6 million USD (Table 3). The annual healthcare cost per patient increased from 813 USD in 2009 to 895 USD in 2013 (Fig. 2).

The number of liver cirrhosis patients was 14,551, which included 5,662 inpatients $(5,662 / 14,551,38.9 \%)$ and 13,887 out-

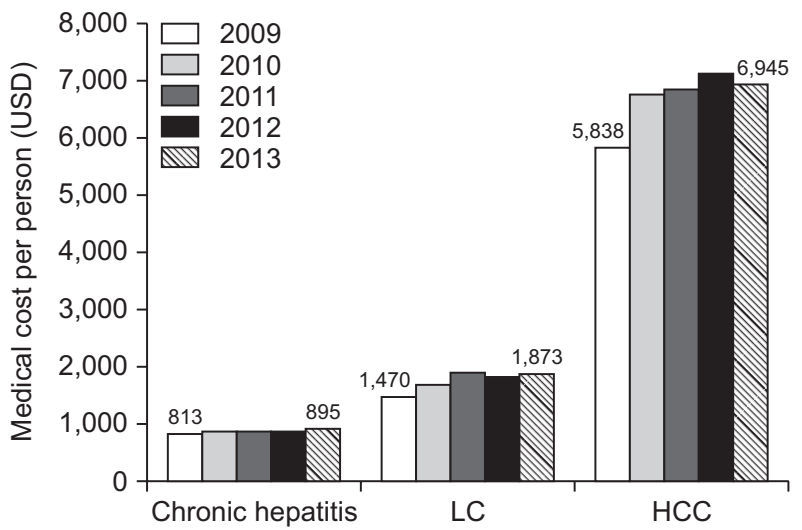

Fig. 2. The healthcare costs per patient for chronic hepatitis $C$ virus infection and related liver diseases by year in South Korea. LC, liver cirrhosis; HCC, hepatocellular carcinoma; USD, United States dollar (1 USD=1,152 South Korean Won). 
patients $(13,887 / 14,551,95.4 \%)$. The overall 5-year cost of the liver cirrhosis patients was 64.6 million USD, which included inpatient and outpatient costs of 35.5 million USD (55\%) and 29.1 million USD (45\%), respectively. The annual cost was 12.9 million USD (Table 2). The annual cost per patient increased from 1,470 USD in 2009 to 1,873 USD in 2013 (Fig. 2).

The number of HCC patients was 7,140 persons, which included 6,552 (6,552/7,140, 91.8\%) inpatients and 6,804 $(6,804 / 7,140,95.3 \%)$ outpatients. The overall 5 -year cost of the HCC patients was 123.0 million USD, which included 87.2 million USD (70.9\%) for inpatient care and 35.8 million USD (29.1\%) for outpatient care. The annual cost was 24.6 million USD (Table 2). The annual cost per patient increased from 5,838 USD in 2009 to 6,945 USD in 2013 (Fig. 2, Supplementary Fig. 1).

For patients in their first year after LT $(n=118)$, the overall 5-year healthcare cost was 7.9 million USD (Table 2) and the annual cost per patient was 67,359 USD, which remarkably decreased after the second year post-LT (Fig. 3, Supplementary Fig. 2).

The detailed cost categories for inpatient services comprised hospital admission fees (26.3\%), injection fees (19.5\%), laboratory test fees (18.4\%), fees for operation or other therapy (13.2\%), and others. On the other hand, the cost categories for outpatient services comprised injection fees (43.7\%), laboratory test fees (28.8\%), and doctor fees (12.6\%) (Supplementary Table 1).

\section{Treatment rate and healthcare costs for interferon-based antiviral therapy}

Between 2009 and 2013, the number of patients who received antiviral treatment including interferons or ribavirin at least once was 25,223, representing $13.9 \%$ of all patients. The total cost associated with antiviral treatment during the 5 years was 138.3 million USD (42.0\% of the total CHC healthcare cost); of these, 99.8 million USD (72.2\%) were pharmaceutical costs. The

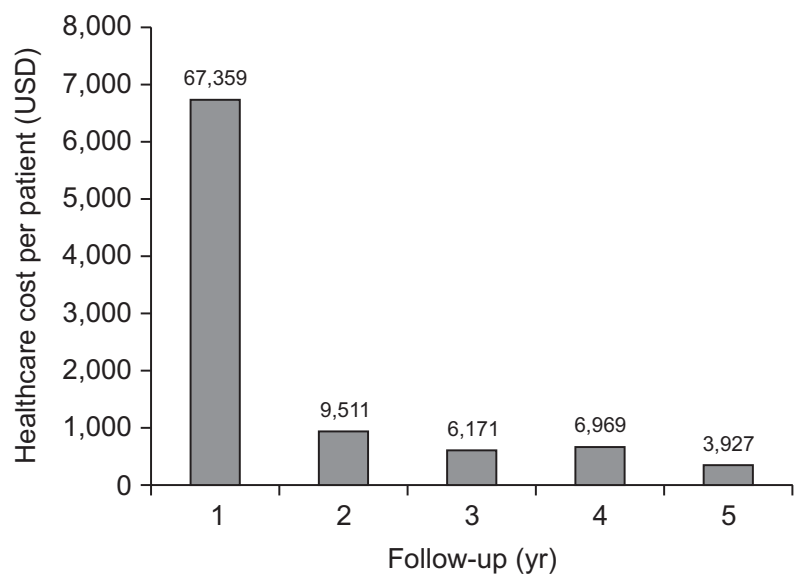

Fig. 3. The healthcare costs per patient in patients undergoing liver transplantation following chronic hepatitis $\mathrm{C}$ virus infection by follow-up year in South Korea. USD, United States dollar ( 1 USD=1,152 South Korean Won).

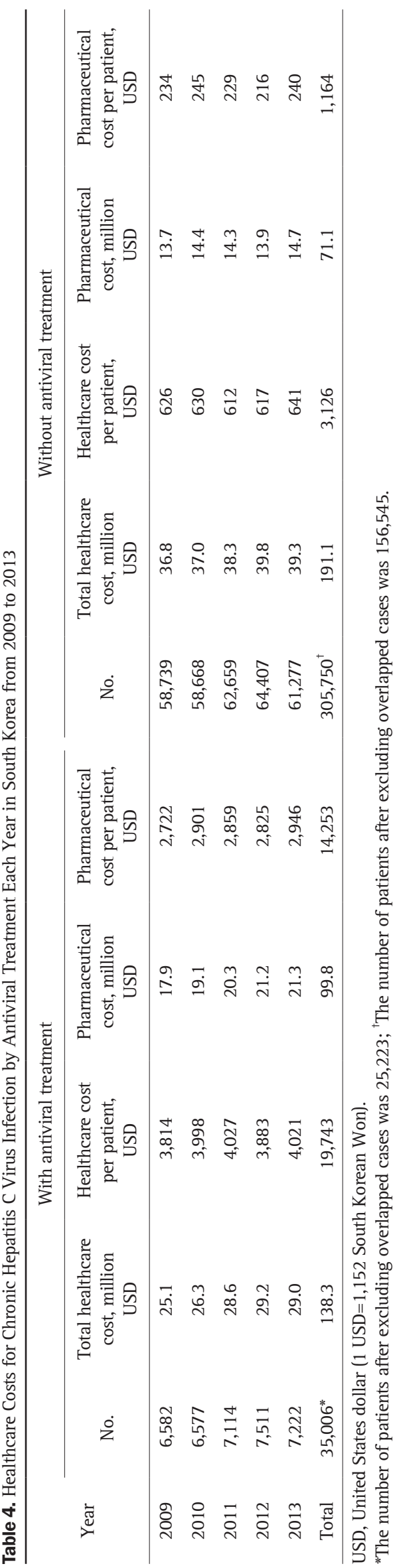


Table 5. Pharmaceutical Costs for Chronic Hepatitis C Virus Infection by Drug Type in South Korea from 2009 to 2013

\begin{tabular}{|c|c|c|c|}
\hline Drug name & No. of patients (\%) & $\begin{array}{l}\text { Pharmaceutical cost, } \\
\text { million USD }\end{array}$ & $\begin{array}{c}\text { Pharmaceutical cost per patient, } \\
\text { USD }\end{array}$ \\
\hline \multicolumn{4}{|c|}{ Patients receiving monotherapy $(n=2,222)$} \\
\hline Interferon- $\alpha-2 \mathrm{a}$ & $56(2.5)$ & 0.03 & 476 \\
\hline Interferon- $\alpha-2 b$ & $49(2.2)$ & 0.07 & 1,355 \\
\hline Peginterferon- $\alpha-2 \mathrm{a}$ & $481(21.7)$ & 1.05 & 2,177 \\
\hline Peginterferon- $\alpha-2 b$ & $165(7.4)$ & 0.34 & 2,041 \\
\hline Ribavirin & $1,471(66.2)$ & 0.32 & 216 \\
\hline Total & $2,222(100)$ & 1.80 & 6,266 \\
\hline \multicolumn{4}{|c|}{ Patients receiving combination therapy $(n=23,001)$} \\
\hline Interferon- $\alpha-2 a+$ ribavirin & $128(0.5)$ & 0.08 & 610 \\
\hline Interferon- $\alpha-2 b+$ ribavirin & $334(1.4)$ & 0.37 & 1,112 \\
\hline Peginterferon- $\alpha-2 a+$ ribavirin & $15,061(64.0)$ & 51.91 & 3,447 \\
\hline Peginterferon- $\alpha-2 b+$ ribavirin & $7,993(34.0)$ & 24.14 & 3,020 \\
\hline Total & $23,516(100)$ & 76.50 & 8,188 \\
\hline
\end{tabular}

USD, United States dollar (1 USD=1,152 South Korean Won).

healthcare cost per patient for the 5-year period was 19,743 USD for those who received antiviral therapy, which was 6.3-fold higher compared to the 3,126 USD for those who did not receive antiviral therapy (Table 4). Of the total 5-year pharmaceutical cost of 99.8 million USD paid for the patients who received antiviral therapy, interferons, peginterferons, and/or ribavirin accounted for 78.3 million USD (78.5\%) (Table 5). Of the 25,223 patients who received antiviral treatment including interferons or ribavirin at least once, 23,001 patients (92.3\%) received combination therapy with peginterferon- $\alpha-2 \mathrm{a}$ or $2 \mathrm{~b}$ and ribavirin, while 2,222 (8.8\%) received monotherapy (Table 5). Among the 156,545 patients who did not receive antiviral therapy, the pharmaceutical costs other than for antiviral therapy accounted for 37.2\% (71.1 million USD) of the total cost (191.1 million USD).

\section{DISCUSSION}

In this study using HIRA claims data from 2009 to 2013, the number of patients with CHC in South Korea over the 5-year period was found to be 181,768 persons, the majority of whom utilized tertiary or general hospital services. Among the total inpatients, 65\%, 16\%, 18\%, and $0.4 \%$ were treated under the diagnoses of chronic hepatitis, liver cirrhosis, HCC, and LT, respectively. In 2013, the annual total healthcare costs per patient for chronic hepatitis, liver cirrhosis, HCC, and LT in the first year were 895, 1,873, 6,945, and 67,359 USD, respectively. The healthcare cost showed a remarkable increase with increasing liver disease severity. Interferon-based antiviral therapeutics, including mostly peginterferons and ribavirin, were prescribed to 25,223 patients for the 5 years, accounting for only $13.9 \%$ of the total CHC patients, thus indicating a highly unmet need for interferon-free antiviral therapy. Taken together, these results indicate an urgent need for treating $\mathrm{CHC}$ before it progresses to advanced liver disease states, and provide valuable baseline data for future analyses of the cost-effectiveness of DAA therapeutics in South Korea.

Although the transmission through blood transfusion has halted since 1990, HCV infection is continuously occurring, owing largely to invasive procedures and the increasing drug abuse in younger generations. Based on a previous study in which 291,000 Koreans aged $\geq 20$ years who received health examinations in 2009 were included, the nationwide age-, sex-, and area-adjusted anti-HCV positive rate reached $0.78 \%{ }^{5}$ However, based on data from the National Health Insurance Service between 2005 to 2012, the prevalence rate of patients aged $\geq 20$ years who received treatment for acute or CHC has been steadily increasing, from $0.14 \%$ in 2005 to $0.18 \%$ in $2012{ }^{6}$

Though the actual number of CHC patients in South Korea is unknown, the current analysis of nationwide HIRA claims data showed that the yearly total numbers of CHC patients were 65,321, 65,245, 69,773, 71,918, and 68,499 in 2009, 2010, 2011, 2012, and 2013, respectively, with a total of 181,768 individual patients for 5 years. Of note, the patient number increased until 2012, after which a decreasing tendency was seen; this trend should be monitored continuously. Moreover, the estimated nationwide anti-HCV prevalence in South Korean adults in 2009 was reported as $0.78 \%,{ }^{5}$ suggesting that about 327,000 (95\% confidence interval, 96,000 to 868,000 ) adults were infected with HCV, of whom approximately 183,000 patients were viremic patients who were indicated for antiviral therapy. ${ }^{5}$ Therefore, only 20\% of CHC patients $(65,321 / 327,000)$ in 2009 may have utilized the healthcare services under a diagnosis of CHC, suggesting a marked underdiagnosis of HCV infection in Korea. 
The unawareness and underdiagnosis of $\mathrm{CHC}$ is a universal phenomenon, ${ }^{7}$ which had led to a birth cohort screening strategy utilized in the United States. ${ }^{8}$

Compared with the study results from a U.S. study reporting that the all-cause healthcare cost of 34,597 CHC patients aged at least 18 years from 2002 to 2013 was 19,665 USD per patient per year, ${ }^{9}$ this study's result of 997 USD per patient per year in 2013 suggests a remarkable difference, likely owing to the different healthcare systems and economic statuses of the countries. Furthermore, in another U.S. study using CHC cohorts, the average lifetime cost of CHC was estimated as 64,490 USD. ${ }^{10}$

In this study, the total healthcare cost markedly increased according to the severity of liver disease, from chronic hepatitis to liver cirrhosis to HCC to LT. The health insurance spending by cancer site reported in 2013 showed that liver cancer was associated with the third highest cost of all cancers, accounting for $10 \%$ of cancer-related health insurance spending. ${ }^{11}$ Additionally, the present study demonstrated that 118 patients, accounting for $0.06 \%$ of all CHC patients, received LT during the 5-year study period. This finding strongly supports the benefit of early treatment before the disease progresses to liver cirrhosis or HCC.

The number of patients who received antiviral therapeutic drugs at least once during the 5 years was only 25,223, with a treatment rate of $13.9 \%$, which is much lower than the previously reported rate of $37.3 \%$ in a Korean study using a CHC cohort. ${ }^{12}$ This gap likely reflects a referral bias related to the highly motivated patients and doctors in university hospitals. In addition, the previous study excluded patients with a previous history of interferon-based therapy and nonviremic patients. ${ }^{12}$ However, our HIRA data have included patients with a sustained viral response after past treatment and spontaneously recovered patients. Nevertheless, this low treatment rate strongly indicates a huge unmet need for interferon-free antiviral therapy. ${ }^{13,14}$ Moreover, our results showed that some patients were prescribed only ribavirin therapy, which is not the standard therapy, and this may reflect an unawareness of doctors to the appropriate HCV antiviral treatment. ${ }^{15,16}$ Therefore, the recently introduced DAA therapy is expected to transform the treatment paradigm of HCV. However, the high drug costs and underdiagnosis of the patients are major hurdles in the cure of HCV infection. ${ }^{17}$

In a U.S. study from 2011, the annual healthcare costs due to HCV-related diseases was reported as 65 billion USD, ${ }^{10}$ and studies about the burden and cost-effectiveness of HCV are currently being performed in Europe, Canada, and Egypt. Although the direct medical costs for liver disease have been reported to have increased from 588.7 billion Korean Won in 2004 to 1,032.2 billion Korean Won in 2008, ${ }^{18}$ neither the medical costs associated with HCV nor the cost-effectiveness of antiviral therapy against HCV has been reported in Korea to date. In the present study, the data were collected through the HIRA database, a nationwide database of medical claims that includes 97\% of all citizens of South Korea; therefore, the data collected were con- sidered representative of the national population.

However, the use of this database also has some limitations. First, ICD-10 codes were used, but the coding accuracy has not been properly evaluated for different disease areas. In addition, the study did not evaluate the clinical outcomes of the patients, because the database contains no variable regarding the clinical outcomes. Moreover, the HIRA database does not collect data on non-reimbursed out-of-pocket costs. These costs were not included in this study, therefore, the real cost would be higher than this result. Another limitation of the present study was that, since this was a retrospective study, there were some missing values and limited information. Finally, the patient population included only those who received medical services for this disease and associated conditions. Therefore, caution is needed when interpreting and generalizing our findings.

In conclusion, the total healthcare costs increased remarkably from chronic hepatitis to liver cirrhosis to HCC to LT patients in Korea. Every effort is needed to stop the disease progression of $\mathrm{CHC}$ and to reduce the HCV-related disease burden. The present results provide baseline information for cost-effectiveness analyses of DAA therapy or for population-based screening strategies in Korea.

\section{CONFLICTS OF INTEREST}

No potential conflict of interest relevant to this article was reported.

\section{ACKNOWLEDGEMENTS}

This work was supported in part by grants from Bristol-Myers Squibb, the Korea Centers for Disease Control and Prevention (grant for the Chronic Infectious Disease Cohort [Korea HCV Cohort Study, 4800-4859-304]), the Korea Health Technology R\&D Project through the Korea Health Industry Development Institute (KHIDI), funded by the Ministry of Health \& Welfare (grant number: HC15C1193) and the National Cancer Center, Korea (NCC-1710141).

\section{REFERENCES}

1. World Health Organization. Global report on access to hepatitis C treatment: focus on overcoming barriers. Geneva: World Health Organization, 2016

2. Nguyen LH, Nguyen MH. Systematic review: Asian patients with chronic hepatitis C infection. Aliment Pharmacol Ther 2013;37:921-936.

3. Kim L, Kim JA, Kim S. A guide for the utilization of Health Insurance Review and Assessment Service National Patient Samples. Epidemiol Health 2014;36:e2014008.

4. Jo C. Cost-of-illness studies: concepts, scopes, and methods. Clin Mol Hepatol 2014;20:327-337. 
5. Kim DY, Kim IH, Jeong SH, et al. A nationwide seroepidemiology of hepatitis C virus infection in South Korea. Liver Int 2013;33:586-594.

6. Shon HS, Choi HY, Kim JR, et al. Comparison and analysis of the prevalence of hepatitis $\mathrm{C}$ virus infection by region in the Republic of Korea during 2005-2012. Clin Mol Hepatol 2015;21:249-256.

7. Cox AL. MEDICINE: global control of hepatitis $C$ virus. Science 2015;349:790-791.

8. Rein DB, Smith BD, Wittenborn JS, et al. The cost-effectiveness of birth-cohort screening for hepatitis $\mathrm{C}$ antibody in U.S. primary care settings. Ann Intern Med 2012;156:263-270.

9. McAdam-Marx C, McGarry LJ, Hane CA, Biskupiak J, Deniz B, Brixner DI. All-cause and incremental per patient per year cost associated with chronic hepatitis $\mathrm{C}$ virus and associated liver complications in the United States: a managed care perspective. J Manag Care Pharm 2011;17:531-546.

10. Razavi H, Elkhoury AC, Elbasha E, et al. Chronic hepatitis C virus (HCV) disease burden and cost in the United States. Hepatology 2013;57:2164-2170.

11. Ministry of Health \& Welfare; National Cancer Center. Cancer facts \& figures 2015. Goyang: National Cancer Center, 2015.

12. Lee SS, Jeong SH, Jang ES, et al. Treatment rate and factors related to interferon-based treatment initiation for chronic hepatitis C in South Korea. J Med Virol 2016;88:275-281.

13. Sun WR, Ju HO. Correlation between knowledge of hepatitis $C$ and compliance with therapeutic guidelines in chronic hepatitis $\mathrm{C}$ patients. J Korean Acad Soc Home Care Nurs 2015;22:69-77.

14. Hsu PC, Federico CA, Krajden M, et al. Health utilities and psychometric quality of life in patients with early- and late-stage hepatitis C virus infection. J Gastroenterol Hepatol 2012;27:149-157.

15. Kim TH, Lee JS. Recent advancement in the treatment of chronic hepatitis C. Korean J Med 2014;86:563-569.

16. Korean Association for the Study of the Liver. KASL clinical practice guidelines: management of hepatitis C. Clin Mol Hepatol 2016;22:76-139.

17. Honegger JR, Zhou Y, Walker CM. Will there be a vaccine to prevent HCV infection? Semin Liver Dis 2014;34:79-88.

18. Lee S, Chung W, Hyun KR. Socioeconomic costs of liver disease in Korea. Korean J Hepatol 2011;17:274-291. 